\title{
Diamond chains with multiple-spin exchange interactions
}

\author{
N. B. Ivanov, ${ }^{1}$ J. Richter ${ }^{2}$ and J. Schulenburg ${ }^{3}$ \\ ${ }^{1}$ Institute of Solid State Physics, Bulgarian Academy of Sciences, Tzarigradsko chaussee 72, 1784 Sofia, Bulgaria \\ ${ }^{2}$ Institut für Theoretische Physik, Universität Magdeburg, PF 4120, D-39016 Magdeburg, Germany \\ ${ }^{3}$ Universitätsrechenzentrum, Universität Magdeburg, D-39016 Magdeburg, Germany
}

(Dated: June 3, 2022)

\begin{abstract}
We study the phase diagram of a symmetric spin- $1 / 2$ Heisenberg diamond chain with additional cyclic four-spin exchange interactions. The presented analysis supplemented by numerical exactdiagonalization results for finite periodic clusters implies a rich phase diagram containing, apart from standard magnetic and spin-liquid phases, two different tetramer-dimer phases as well as an exotic four-fold degenerate dimerized phase. The characteristics of the established spin phases as well as the nature of quantum phase transitions are discussed, as well.
\end{abstract}

PACS numbers: $75.10 . \mathrm{Pq}, 75.30 . \mathrm{Kz}, 75.40 . \mathrm{Mg}$

\section{INTRODUCTION}

Spin systems with cyclic exchange interactions have been receiving an increasing amount of attention in the past few years. These interactions are known to be responsible for the properties of the triangular magnetic system composed of ${ }^{3} \mathrm{He}$ atoms absorbed on graphite surfaces. $\underline{\underline{1}}$ Recently, it was demonstrated that a certain amount of four-spin exchange could explain the neutron-scattering experiments concerning high- $T_{c}$ compounds such as $\mathrm{La}_{2} \mathrm{CuO}_{4} \stackrel{2}{,}, \mathrm{La}_{6} \mathrm{Ca}_{8} \mathrm{Cu}_{2} 4 \mathrm{O}_{41} \stackrel{3}{,}$, and $\mathrm{La}_{4} \mathrm{Sr}_{10} \mathrm{Cu}_{24} \mathrm{O}_{41} \stackrel{4}{*}$ The latter two are spin-ladder compounds where charge fluctuations such as cyclic hopping processes modify the Heisenberg spin Hamiltonian by contributing a four-spin interaction term. In particular, it was found that these interactions substantially modify the spin triplon gaps and frustrate the formation of bound triplon states. $\frac{4}{1}$ On the theoretical side, in spite of the numerous numerical studies predicting rich phase diagrams,$\frac{5}{5}$ a number of important questions, concerning the type of spin orderings and quantum criticalities realized by the four-spin exchange, remain unsettled. 6

In this paper we analyze the role of the cyclic four-spin exchange interactions in the symmetric diamond chain (SDC) which is one of the simplest spin models admitting multispin cyclic exchange interactions. The model is defined by the Hamiltonian (see Fig. 1)

$$
\mathcal{H}=\sum_{n=1}^{L} h_{n, n+1}
$$

where

$$
\begin{aligned}
h_{n, n+1} & =J_{1} \mathbf{s}_{n} \cdot\left(\boldsymbol{\sigma}_{n}+\boldsymbol{\sigma}_{n+1}\right)+J \boldsymbol{\sigma}_{n} \cdot \boldsymbol{\sigma}_{n+1} \\
& +J_{\perp} \mathbf{s}_{\mathbf{1} n} \cdot \mathbf{s}_{\mathbf{2} n}+K\left[\left(\mathbf{s}_{\mathbf{1} n} \cdot \boldsymbol{\sigma}_{n}\right)\left(\mathbf{s}_{\mathbf{2} n} \cdot \boldsymbol{\sigma}_{n+1}\right)\right. \\
& \left.+\left(\mathbf{s}_{\mathbf{1} n} \cdot \boldsymbol{\sigma}_{n+1}\right)\left(\mathbf{s}_{\mathbf{2} n} \cdot \boldsymbol{\sigma}_{n}\right)-\left(\mathbf{s}_{\mathbf{1} n} \cdot \mathbf{s}_{\mathbf{2} n}\right)\left(\boldsymbol{\sigma}_{n} \cdot \boldsymbol{\sigma}_{n+1}\right)\right] .
\end{aligned}
$$

Here $\mathbf{s}_{\mathbf{1}_{n}}, \mathbf{s}_{\mathbf{2}}$, and $\boldsymbol{\sigma}_{n}$ are spin-1/2 operators defined on the sites of the $n$-th elementary cell. In our model, the standard cyclic four-spin exchange interactions ${ }^{7}$ are slightly generalized by including its bilinear terms in the exchange parameters $J, J_{1}$, and $J_{\perp}$. In what follows the action and the energy are measured in the units of $\hbar$ and $J_{1}$, respectively.

The frustrated diamond chain ${ }^{8}$ as well as its various modifications, such as the distorted diamond chain 9,10 and the so-called $A B_{2}$ ferrimagnetic chain with Ising and Heisenberg spins ${ }^{11}$, have already been discussed in the literature in relation to some quasi-1D magnetic materials 12 For the following analysis it is important to notice that the cyclic exchange interaction does not violate the local symmetry of the Hamiltonian under the exchange of the pair of spins $\left(\mathbf{s}_{\mathbf{1}_{n}}, \mathbf{s}_{\mathbf{2}}\right)$ for each diamond in the SDC. Thus, in the important case of spin-1/2 operators $\mathbf{s}_{\mathbf{1}_{n}}$ and $\mathbf{s}_{\mathbf{2}_{n}}$, the system is characterized by $L$ local good quantum numbers $s_{n}=0,1(n=1,2, \ldots, L)$ related to the composite spins $\mathbf{s}_{n}=\mathbf{s}_{\mathbf{1}_{n}}+\mathbf{s}_{\mathbf{2}_{n}}: \mathbf{s}_{n}^{2}=$ $s_{n}\left(s_{n}+1\right)$. Using this local symmetry and the standard commutation relations for spin operators, the local Hamiltonian $h_{n, n+1}$ can be represented in the compact form

$$
\begin{aligned}
h_{n, n+1} & =\epsilon_{n}+\mathbf{s}_{n} \cdot\left(\boldsymbol{\sigma}_{n}+\boldsymbol{\sigma}_{n+1}\right)+J_{n} \boldsymbol{\sigma}_{n} \cdot \boldsymbol{\sigma}_{n+1} \\
& +\frac{K}{2}\left\{\mathbf{s}_{n} \cdot \boldsymbol{\sigma}_{n}, \mathbf{s}_{n} \cdot \boldsymbol{\sigma}_{n+1}\right\} .
\end{aligned}
$$

Here $\epsilon_{n} / J_{\perp}=s_{n}\left(s_{n}+1\right) / 2-3 / 4$ are fixed numbers $(-3 / 4$ or $1 / 4)$ for every sector defined as a sequence of the local quantum numbers $\left[s_{1}, s_{2}, \ldots, s_{L}\right], J_{n}=J+K / 4-$ $s_{n}\left(s_{n}+1\right) K / 2$, and $\{A, B\}$ is the anticommutator of two operators $(A$ and $B)$.

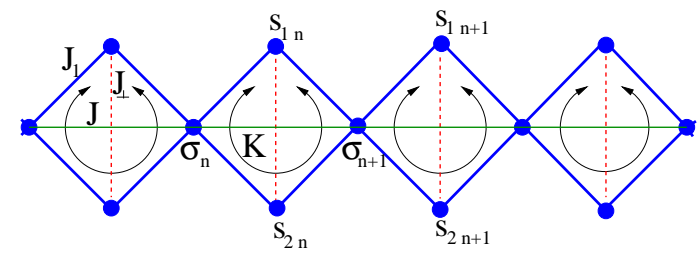

FIG. 1: The symmetric diamond chain considered in the paper. The arrows denote the cyclic four-spin exchange interaction controlled by the parameter $K$.

In the parameter space where the ground state is characterized by $s_{n}=0(n=1,2, \ldots, L)$, the second and 
fourth terms in Eq. (2) vanish and the model is equivalent to the spin- $1 / 2$ Heisenberg chain with an exchange parameter $J+K / 4$. On the other hand, if the ground state belongs to the sector $s_{n}=1(n=1,2, \ldots, L)$, Eq. (2) defines the generic model of an alternating mixed-spin chain with the simplest three-spin exchange interaction. Finally, if the ground state is in the sector $[1,0,1,0, \ldots]$, the system is reduced to some modification of the wellknown orthogonal dimer chain. 13

\section{PHASE DIAGRAM IN THE PARAMETER $\operatorname{SPACE}\left(K, J_{\perp}\right)$}

It is instructive to begin with the phase diagram of a single diamond composed of the spins $\mathbf{s}_{\mathbf{1}}, \mathbf{s}_{\mathbf{2}}, \boldsymbol{\sigma}_{1}$, and $\boldsymbol{\sigma}_{2}$. The diamond eigenstates (see Table 1) consist of two singlets $\left(S_{1}\right.$ and $\left.S_{2}\right)$, three triplets $\left(T_{1}^{\mu}, T_{2}^{\mu}\right.$, and $\left.T_{3}^{\mu} ; \mu=0, \pm 1\right)$, and the quintet $Q^{\mu}(\mu=0, \pm 1, \pm 2)$. In Figure 2, the phase boundaries between the singlediamond ground states $\left(S_{1}, S_{2}, T_{1}\right.$, and $\left.T_{2}\right)$ are depicted by dashed lines. As discussed below, some of these lines coincide with the exact phase boundaries of the diamond chain, Eq. (1).

\begin{tabular}{l|lll|l|l}
\hline & $s_{p}$ & $s$ & $\sigma$ & \multicolumn{1}{|c|}{ Eigenvalue } & \multicolumn{1}{|c}{ Eigenstate } \\
\hline$S_{1}$ & 0 & 0 & 0 & $-\frac{3}{4}\left(J_{\perp}+J\right)-\frac{3}{16} K$ & $t_{\perp}^{s} t_{\|}^{s}$ \\
$S_{2}$ & 0 & 1 & 1 & $-2+\frac{1}{4}\left(J_{\perp}+J\right)+\frac{13}{16} K$ & $\frac{1}{\sqrt{3}}\left(t_{\perp}^{+} t_{\|}^{-}+t_{\perp}^{-} t_{\|}^{+}-t_{\perp}^{0} t_{\|}^{0}\right)$ \\
$T_{1}^{0}$ & 1 & 1 & 1 & $-1+\frac{1}{4}\left(J_{\perp}+J\right)-\frac{7}{16} K$ & $\frac{1}{\sqrt{2}}\left(t_{\perp}^{+} t_{\|}^{-}-t_{\perp}^{-} t_{\| \mid}^{+}\right)$ \\
$T_{2}^{\mu}$ & 1 & 0 & 1 & $-\frac{3}{4} J_{\perp}+\frac{J}{4}+\frac{K}{16}$ & $t_{\perp}^{s} t_{\| \mid}^{\mu}, \quad \mu=0, \pm$ \\
$T_{3}^{\mu}$ & 1 & 1 & 0 & $\frac{J_{\perp}}{4}-\frac{3}{4} J+\frac{K}{16}$ & $t_{\perp}^{\mu} t_{\| \mid}^{s}, \quad \mu=0, \pm$ \\
$Q^{0}$ & 2 & 1 & 1 & $1+\frac{1}{4}\left(J_{\perp}+J\right)+\frac{K}{16}$ & $\frac{1}{\sqrt{6}}\left(t_{\perp}^{+} t_{\|}^{-}+t_{\perp}^{-} t_{\|}^{+}+2 t_{\perp}^{0} t_{\| \mid}^{0}\right)$
\end{tabular}

TABLE I: Eigenvalues and eigenstates of a single diamond composed of the spins $\mathbf{s}_{1}, \mathbf{s}_{2}, \boldsymbol{\sigma}_{1}$, and $\boldsymbol{\sigma}_{2}$. The eigenstates are classified according to the following good quantum numbers of the single-diamond cluster: the total diamond spin $s_{p}$, its $z$ component $s_{p}^{z}$, and the diagonal spins $s$ and $\sigma\left[\mathbf{s}=\mathbf{s}_{1}+\mathbf{s}_{2}\right.$ and $\left.\boldsymbol{\sigma}=\boldsymbol{\sigma}_{1}+\boldsymbol{\sigma}_{2}: \mathbf{s}^{2}=s(s+1), \boldsymbol{\sigma}^{2}=\sigma(\sigma+1)\right]$. The symbols $t^{\mu}$ $(\mu=0, \pm)$ stand for the canonical basic states of the spin-1 operators, whereas $t^{s}$ denotes the singlet state of two spin$1 / 2$ operators. For brevity, only the $s_{p}^{z}=0$ components of the triplet $\left(T_{1}^{\mu}\right)$ and quintet $\left(Q^{\mu}\right)$ states are presented.

\section{A. Phases in the sector $[0,0, \cdots, 0]$}

For large enough values of the parameter $J_{\perp}$, the offchain spins form local dimers, $\mathbf{s}_{\mathbf{1}_{n}} \cdot \mathbf{s}_{\mathbf{2}_{n}}=-3 / 4$ so that the ground state of the model belongs to the sector $[0,0, \cdots, 0]$. Thus, Eq. (2) reduces to the form

$$
h_{n, n+1}=-(3 / 4) J_{\perp}+(J+K / 4) \boldsymbol{\sigma}_{n} \cdot \boldsymbol{\sigma}_{n+1} .
$$

This is the Hamiltonian of a spin-1/2 Heisenberg chain with the exchange constant $J+K / 4$. The single-diamond line $A B$ (defined by $J+K / 4=0$ ) coincides with the exact boundary between the fully polarized ferromagnetic phase FM1 $(J+K / 4<0)$ and the critical spin-fluid phase SF $(J+K / 4>0)$.

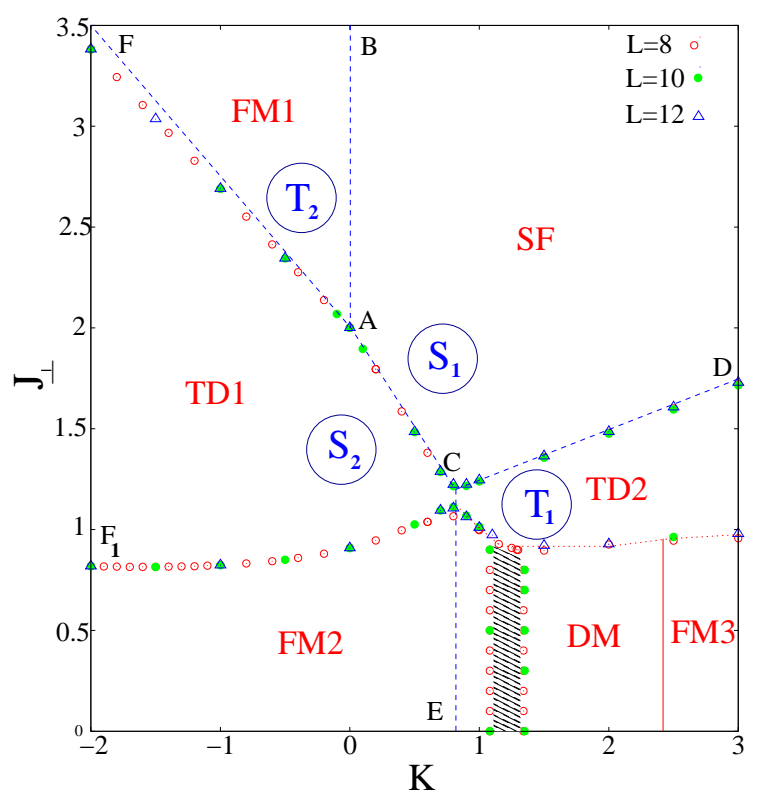

FIG. 2: Phase diagram of the model, Eq. (1), in the parameter space $\left(K, J_{\perp}\right)$ for $J=0$ as obtained from the numerical diagonalization of periodic clusters containing up to $L=12$ unit cells. The dashed lines denote the boundaries of the single-diamond phase diagram containing the states $S_{1}, S_{2}$, $T_{1}$, and $T_{2}$ (see Table 1 ). The following abbreviations for the phases of Eq. (1) are used: critical spin-fluid state (SF), fully-polarized ferromagnetic state (FM1), ferrimagnetic state (FM2), tetramer-dimer states (TD1,TD2), dimerized singlet state (DM), and another ferrimagnetic state (FM3).

Besides the well-documented collective modes, these phases exhibit additional single-particle modes (related to the off-diagonal spins) describing excited $\left(\mathbf{s}_{\mathbf{1}_{n}}, \mathbf{s}_{\mathbf{2}_{n}}\right)$ dimers, $s_{n}=1$. Being eigenstates of the Hamiltonian, Eq. (1), these excitations are completely localized. According to Eq. (2), an excited dimer (say at $n=L$ ) is described by the Hamiltonian

$$
\mathcal{H}_{i}=C+\left(J+\frac{K}{4}\right) \sum_{n=1}^{L-1} \boldsymbol{\sigma}_{n} \cdot \boldsymbol{\sigma}_{n+1}+h_{i}
$$

where $C=-\frac{3}{4} J_{\perp}(L-1)$ and $h_{i}$ reads

$$
\begin{aligned}
h_{i} & =\frac{J_{\perp}}{4}+\left(J-\frac{3 K}{4}\right) \boldsymbol{\sigma}_{L} \cdot \boldsymbol{\sigma}_{1}+J_{i} \mathbf{s}_{L} \cdot\left(\boldsymbol{\sigma}_{L}+\boldsymbol{\sigma}_{1}\right) \\
& +\frac{K}{2}\left\{\mathbf{s}_{L} \cdot \boldsymbol{\sigma}_{L}, \mathbf{s}_{L} \cdot \boldsymbol{\sigma}_{1}\right\}, J_{i}=1 .
\end{aligned}
$$

Similar models, describing spin- $S$ impurities in spin- $1 / 2$ antiferromagnetic Heisenberg chains, have been discussed in the literature. 14 In particular, the above model may be considered as a special class of open chains with symmetric couplings of the end spins $\left(\boldsymbol{\sigma}_{1}\right.$ and $\left.\boldsymbol{\sigma}_{L}\right)$ to the external spin $\mathbf{s}_{L}\left(s_{L}=1\right)$.

Turning to the region occupied by the SF phase, renormalization-group arguments predict that symmetric perturbations to the open chain are, at most, 
marginal. Thus, the marginally relevant perturbation $J_{i} \mathbf{s}_{L} \cdot\left(\boldsymbol{\sigma}_{L}+\boldsymbol{\sigma}_{1}\right)\left(J_{i}>0\right)$ is expected to renormalize to $\infty$ in the SF phase. This corresponds to a fixed point where the end spins $\boldsymbol{\sigma}_{1}$ and $\boldsymbol{\sigma}_{L}$ are effectively removed from the chain to screen the external spin $\mathbf{s}_{L}$. In terms of the original model, Eq. (11), the spins $\boldsymbol{\sigma}_{1}, \boldsymbol{\sigma}_{L}, \mathbf{s}_{\mathbf{1}_{L}}$, and $\mathbf{s}_{\mathbf{2} L}$ form a decoupled single-diamond state $\left(S_{2}\right)$ in the low-energy sector of the spectrum. Such local excitations are relevant relatively close to the phase boundary $A B$, where the coupling $J_{i} \mathbf{s}_{L} \cdot\left(\boldsymbol{\sigma}_{L}+\boldsymbol{\sigma}_{1}\right)$ dominates the biquadratic term in Eq. (5).

For larger values of the parameter $K$, the biquadratic exchange in $h_{i}$ becomes important. Since the energy of the quintet state $Q$ grows up with $K$ (see Table 1), we concentrate on the triplet state $T_{1}$ whose energy decreases with $K$ and crosses the energy level of $S_{2}$ at $K=4 / 5$. As discussed in Ref. 14, such a local triplet state does not correspond to a stable fixed point, since the antiferromagnetic interaction of the effective spin-1 impurity with the rest of the chain is marginally relevant. Thus, one expects that the couplings to the next two spins in the chain, $\boldsymbol{\sigma}_{L-1}$ and $\boldsymbol{\sigma}_{2}$, grow up to $\infty$. There appears another fixed point where the latter two spins also decouple from the chain in order to screen the spin of the triplet state $T_{1}$. Note that in sectors containing more spin-1 dimers, other types of screening are possible, as well. For example, the spins of neighboring $T_{1}$ diamonds in Fig. 3 may be screened by forming a singlet state. As before, the number of decoupled chain spins is four. We suggest that such decoupled singlet states, composed of longer $S_{2}$ or $T_{1}$ diamond chains, control the observed instability of the SF phase upon decreasing the parameter $J_{\perp}$. Note that similar complexes of $S_{1}$ diamonds can not produce the instability, since the energy of the resulting product state $\left[\boldsymbol{\sigma}_{1}, \boldsymbol{\sigma}_{2}\right]\left[\boldsymbol{\sigma}_{3}, \boldsymbol{\sigma}_{4}\right] \cdots\left[\boldsymbol{\sigma}_{L-1}, \boldsymbol{\sigma}_{L}\right]$ exceeds the energy of the critical phase for arbitrary $J_{\perp}, K>0$.

\section{B. Tetramer-dimer phases}

A detailed numerical study of periodic clusters containing up to $L=12$ cells close to the lines $F A, A C$, and $C D$ in Fig. 2 suggests that for smaller values of $J_{\perp}$ the ground state belongs to the sector $[1,0, \cdots, 1,0]$. In the special case $K=0$, our numerical results reproduce the phase diagram of the frustrated SDC,,$\frac{8}{}$ where the socalled tetramer-dimer phase (denoted as TD1 in Fig. 2) appears in the interval $0.909<J_{\perp}<2$. This doubly degenerate singlet state may be roughly represented as a product of single-diamond $S_{2}$ states on every second diamond, as depicted in Fig. 3. The short-range correlations shown in Fig. 3 imply that the simple product state is a good variational wave function over the entire region occupied by the TD1 phase, excluding a narrow region near the line $\mathrm{AE}$. The product state is an exact ground state at $\left(K, J_{\perp}\right)=(0,2)$. An extrapolation of the exact-diagonalization (ED) data suggests that the phase boundary between the TD1 and SF phases lies extremely close to the $A C$ line separating the single-diamond states $S_{1}$ and $S_{2}$. On the other hand, the phase boundary between the TD1 and FM1 phases clearly deviates from the single-diamond boundary AF. The deviation from the line $\mathrm{AF}$ is related to weak but finite interactions between neighboring $S_{2}$ diamonds appearing in the second-order perturbation theory in the parameter $J+K / 4$.

On increasing the cyclic exchange parameter $K$ at fixed $J_{\perp}$ one finds another tetramer-dimer type state (TD2). The picture of the spin-spin correlations, Fig. 3, suggests that every second diamond is approximately in the $T_{1}$ state. Nevertheless, the numerical analysis shows relatively strong antiferromagnetic correlations between neighboring $T_{1}$ diamonds, as opposed to the TD1 state where the $S_{2}$ diamonds are weakly correlated. Clearly, both tetramer-dimer phases are gapped and doublydegenerated. According to the general rules,,$\stackrel{15}{\leftrightarrows}$ one may expect a discontinuous quantum phase transition between the quantum gapped phases TD1 and TD2. Numerically, the transition point is indistinguishable from the exact single-diamond phase boundary $C E(K=4 / 5)$ separating the single-diamond states $S_{2}$ and $T_{1}$.

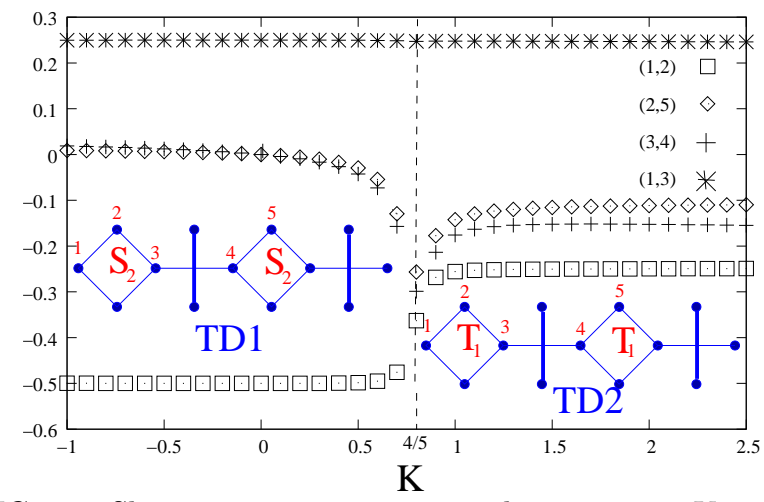

FIG. 3: Short-range spin-spin correlations vs. $K$ in the tetramer-dimer phases TD1 and TD2 for periodic chains with $L=8$ cells $\left(J=0, J_{\perp}=1.15\right) .(1,2) \equiv\left\langle\boldsymbol{\sigma}_{n} \cdot \boldsymbol{s}_{\mathbf{1}_{n}}\right\rangle,(1,3) \equiv$ $\left\langle\boldsymbol{\sigma}_{n} \cdot \boldsymbol{\sigma}_{n+1}\right\rangle,(3,4) \equiv\left\langle\boldsymbol{\sigma}_{n+1} \cdot \boldsymbol{\sigma}_{n+2}\right\rangle,(2,5) \equiv\left\langle\boldsymbol{s}_{\mathbf{1}_{n}} \cdot \boldsymbol{s}_{\mathbf{1}_{n+2}}\right\rangle$.

\section{Phases in the sector $[1,1, \cdots, 1]$}

A numerical inspection of the short-range correlators $\left\langle\mathbf{s}_{\mathbf{1}_{n}} \cdot \mathbf{s}_{\mathbf{2}_{n}}\right\rangle$ in finite periodic chains implies that for moderate values of the parameter $J_{\perp}$ the established ground states belong to the sector $[1,1, \cdots, 1]$. Thus, in the lowenergy region the diamond model, Eqs. (11) and (2), is equivalent to the following mixed-spin Heisenberg model $\left(\left|\mathbf{s}_{n}\right| \equiv s_{1}=1,\left|\boldsymbol{\sigma}_{n}\right| \equiv s_{2}=1 / 2\right)$ with three-spin exchange interactions

$$
\begin{aligned}
\mathcal{H}_{1} & =\sum_{n=1}^{L}\left[\mathbf{s}_{n} \cdot\left(\boldsymbol{\sigma}_{n}+\boldsymbol{\sigma}_{n+1}\right)-J^{\prime} \boldsymbol{\sigma}_{n} \cdot \boldsymbol{\sigma}_{n+1}\right. \\
& \left.+\frac{K}{2}\left\{\mathbf{s}_{n} \cdot \boldsymbol{\sigma}_{n}, \mathbf{s}_{n} \cdot \boldsymbol{\sigma}_{n+1}\right\}\right]
\end{aligned}
$$


where $J^{\prime}=3 K / 4-J$. To the best of our knowledge, generic mixed-spin quantum Heisenberg models with multiple-spin exchange interactions have not been discussed in the literature, although these interactions may play an important role in some recently synthesized mixed-spin magnetic materials $\frac{16.17}{}$ Here we restrict ourselves to a general overview of the spin phases in the specific case $\left(s_{1}, s_{2}\right)=(1,1 / 2)$, and to ferromagnetic exchange interactions between the $\boldsymbol{\sigma}_{n}$ spins $\left(J^{\prime}>0\right)$. The first two terms in Eq. (6) define a standard mixed-spin Heisenberg model containing additional non-frustrated $J^{\prime}$ exchange bonds. The Lieb-Mattis theorem 18 predicts a ferrimagnetic ground state for this bipartite model which coincides with the classical ferrimagnetic twosublattice Néel state (the FM2 phase in Fig. 2). To study the role of the competing three-spin interactions, we use a qualitative spin-wave analysis supplemented by numerical ED calculations for finite periodic chains.
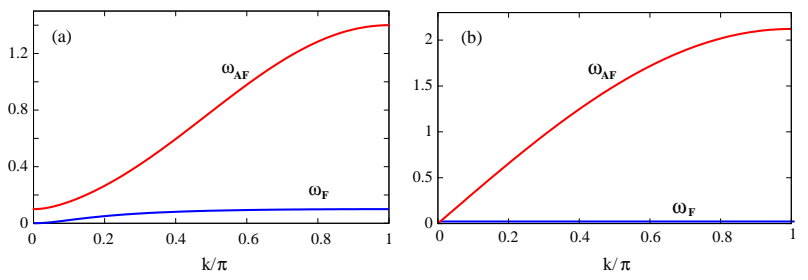

FIG. 4: Spin-wave spectrum of the $\left(s_{1}, s_{2}\right)=(1,1 / 2)$ chain with three-spin exchange interactions, Eq. (6), in the ferrimagnetic (a) and the canted (b) ground states at $\kappa=0.8$ and 1.5 , respectively. $J=J_{\perp}=0, \kappa \equiv K / 2$.

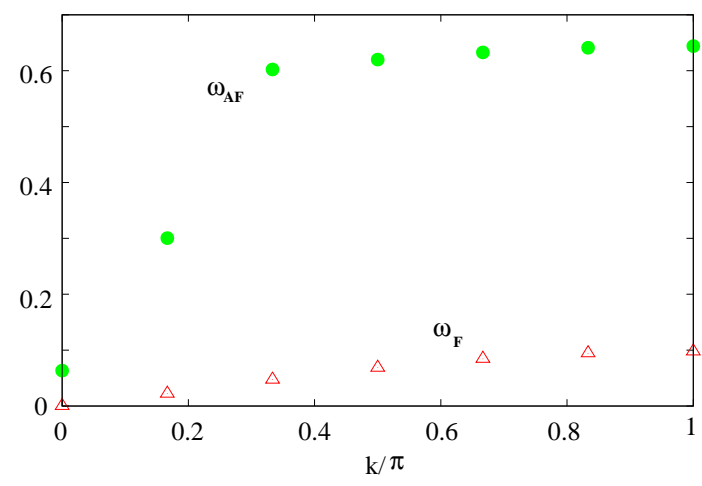

FIG. 5: Lowest-energy excited states in a periodic chain with $L=12$ diamonds in the sectors with magnetic moments $M_{0}-$ 1 (triangles) and $M_{0}+1$ (circles). $M_{0}=L\left(S_{1}-S_{2}\right)=6$ is the magnetic moment of the ferrimagnetic ground state. $K=1$, $J=J_{\perp}=0$.

Let us start with a discussion of the semi-classical limit of the model (6). Since the three-spin interaction contains an additional overall factor of $s_{1} s_{2}$, it is convenient to redefine the coupling constant $K$ and measure the strength of these interactions in terms of $\kappa=s_{1} s_{2} K$. The classical Néel configuration defined by $\mathbf{s}_{n}=s_{1}(0,0,1)$ and $\boldsymbol{\sigma}_{n}=s_{2}(0,0,-1)$ survives up to $\kappa=1$. For $\kappa>1$, the ferromagnetic arrangement of the smaller $s_{2}$ spins remains unchanged whereas the orientation of the $s_{1}$ spins deviates from the $\mathrm{z}$ axis: $\mathbf{s}_{n}=s_{1}\left(\sin \theta \cos \phi_{n}, \sin \theta \sin \phi_{n}, \cos \theta\right)$. Here $\cos \theta=1 / \kappa$ and the azimuthal angle $\phi_{n}$ takes arbitrary values from the interval $0 \leq \phi_{n}<2 \pi(n=1, \ldots, L)$. As a rule, quantum fluctuations favor the planar spin configurations $\left(\phi_{n}=0, \pi ; n=1,2, \ldots, L\right)$, so that one may expect a reduction of the degeneracy to $2^{L}$. Such high degeneracy of the ground state is typical for a number of spin models on corner-sharing lattices. As seen in Fig. 4b, in a spin-wave approximation the degeneracy produces a full line of zero modes $\omega_{F}=0$ in the Brillouin zone. Note that the presence of the gapless antiferromagnetic mode $\omega_{A F} \propto k(k \ll 1)$ for $\kappa>1$ is related to the finite transverse magnetization of the classical canted state. The explicit expression for $\omega_{A F}$ reads

$$
\omega_{A F}=2 J^{\prime} s_{2}\left|\sin \left(\frac{k}{2}\right)\right| \sqrt{\sin ^{2}\left(\frac{k}{2}\right)+\frac{s_{1}}{s_{2}} \alpha \cos k},
$$

where $\alpha=\left(\kappa^{2}-1\right) /\left(2 \kappa J^{\prime}\right)$. It is clear that the antiferromagnetic mode $\omega_{A F}$ is stabilized by the ferromagnetic couplings between the $\boldsymbol{\sigma}_{n}$ spins. The above picture of low-lying excitations in the ferrimagnetic phase close to the phase transition point is confirmed by the numerical

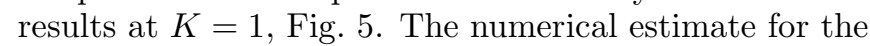
phase transition point is $K_{c} \approx 1.2$.
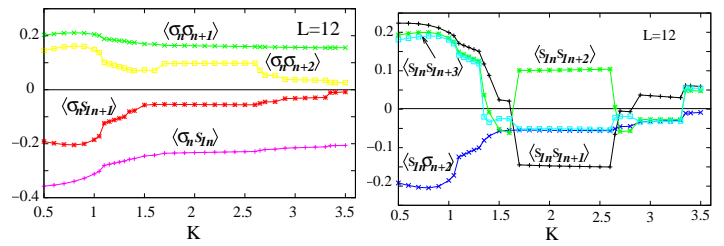

FIG. 6: Short-range spin-spin correlations vs. $K$ for periodic chains with $L=12$ cells $\left(J=J_{\perp}=0\right)$.

Additional information about the phase diagram of Eq. (6) may be obtained from the behavior of the shortrange correlations, Fig. 6, and the singlet-triplet excitation gap, Fig. 7, with the parameter $K$. For larger $K>1.2$, the ED results indicate at least two additional phases denoted by DM and FM3 in Fig. 2. DM is a nonmagnetic gapped singlet state stabilized approximately in the region $1.5 \leq K \leq 2.3$ (see Fig. 7), and FM3 is a magnetic phase similar to the FM2 phase. Since the unit cell consists of three spin- $1 / 2$ variables, the Lieb-Schultz-Mattis theorem ${ }^{19}$ suggests that the spin gap must be accompanied with at least doubly-degenerate ground states.

Further information about the DM phase can be extracted from the dimerization operator $D_{n}=\mathbf{S}_{n} \cdot \mathbf{S}_{n+1}-$ $\mathbf{S}_{n} \cdot \mathbf{S}_{n-1}$ where $\mathbf{S}_{n}$ is the spin operator at site $n$. The lattice sites $n-1$ and $n+1$ are supposed to be symmetric 
under the reflection from the central site $n$. It is convenient to use the symmetric (antisymmetric) combinations $|s, a\rangle=(|0\rangle \pm|1\rangle) / \sqrt{2}$, where $|0\rangle$ is the translationally invariant singlet ground state of the finite periodic chain and $|1\rangle$ is the singlet excited state which is almost degenerate with $|0\rangle$. The states $|s, a\rangle$ are not translationally invariant and may be expected to produce finite values of the dimer order-parameter $\left\langle s, a\left|D_{n}\right| s, a\right\rangle$ in a dimerized system.20 The caricature of the DM state presented in Fig. 8 is obtained from the extrapolation of the ED results for $\left\langle s, a\left|D_{n}\right| s, a\right\rangle(L=8,10$, and 12). A pronounced enhancement of the dimer order-parameter with $L$ is indicated only for two types of bonds in the dimer model (the thick lines in Fig. 8). Clearly, the suggested ground state exhibits a four-fold degeneracy.

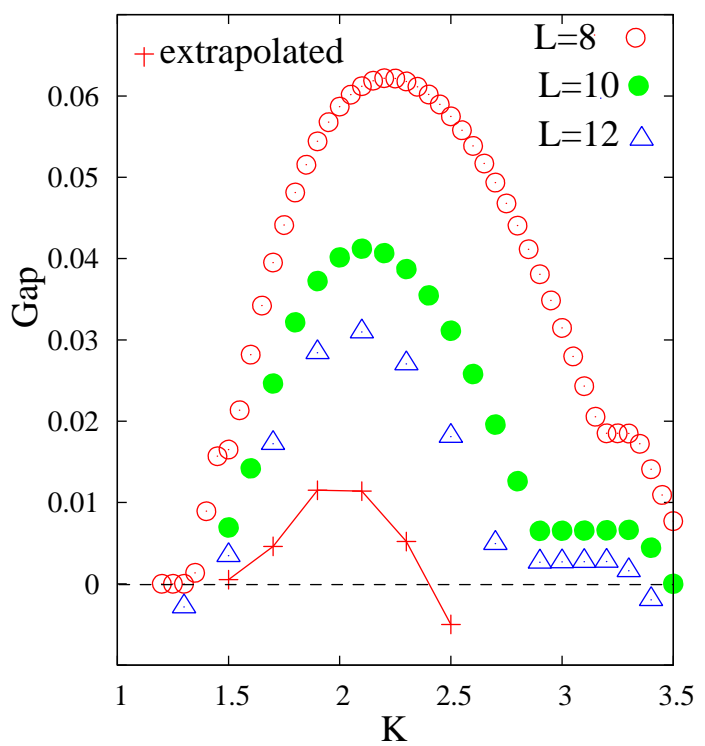

FIG. 7: The excitation gap in the DM phase as a function of the parameter $K$.

Finally, the numerical results point towards the existence of another partially polarized magnetic phase in the narrow interval $1.2<K<1.5$ between the ferrimagnetic (FM2) and the dimerized (DM) phases (the hatched area in Fig. 2). Recently, similar exotic magnetic states have been predicted in a number of one-dimensional spin systems with magnetic frustrations $\stackrel{21}{2}$ Typically, discussed spin states exhibit a partially polarized magnetization in the $z$ direction $\left(M<M_{0}=s_{1}-s_{2}\right)$, a quasi-long-range transverse magnetic order, and a gapless linear mode related to the destroyed classical canted state. Unfortunately, the methods used in the present study do not suggest a clear statement indicating the existence of such an exotic phase in the discussed system.

\section{SUMMARY}

In conclusion, we have examined the impact of the cyclic four-spin exchange interactions on the groundstate phase diagram of the symmetric spin- $1 / 2$ diamond chain. Using the local symmetries of the model, the spin

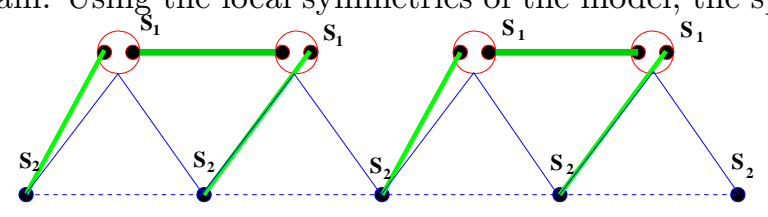

FIG. 8: Caricature of the dimerized singlet phase DM as obtained from an extrapolation of the numerical ED results for periodic chains with $L=8,10$, and 12 . The open circles (thick lines) denote the symmetric (antisymmetric) eigenstates of two spin- $1 / 2$ operators. $K=2, J_{\perp}=J=0$, $\left(s_{1}, s_{2}\right)=(1,1 / 2)$.

phases were classified by the set of good quantum numbers $s_{n}=0,1(n=1,2, \ldots, L)$ related to the composite spins $\mathbf{s}_{n}=\mathbf{s}_{\mathbf{1}_{n}}+\mathbf{s}_{\mathbf{2}_{n}}$. The presented study demonstrates a rich phase diagram in the parameter space $\left(K, J_{\perp}\right)$. Apart from the standard magnetic and paramagnetic phases, the system exhibits two different tetramer-dimer phases in the sector $[1,0,1,0, \cdots, 1,0]$ as well as an exotic four-fold-degenerate dimerized ground state in the sector $[1,1, \cdots, 1]$.

\section{Acknowledgments}

This research was supported by Deutsche Forschungsgemeinschaft (Grant 436 BUL 17/9/06) and the Bulgarian Science Foundation (Grants F1414 and D002264/18.12.08). Part of the work has been done in MaxPlanck-Institut für Physik komplexer Systeme, Dresden.
1 M. Roger, J. H. Hetherington, and J. M. Delrieu, T. Manabe, and M. Yamashita, Rev. Mod. Phys. 55, 1 (1983).

2 R. Coldea, S. M. Hayden, G. Aeppli, T. G. Perring, C. D. Frost, T. E. Mason, S. W. Cheong, and Z. Fisk, Phys. Rev. Lett. 86, 5377 (2001).

3 S. Brehmer, H.-J. Mikeska, M. Müller, N. Nagaosa, and S. Uchida, Phys. Rev. B 60, 329 (1999); M. Matsuda, K. Katsumata, R. S. Eccleston, S. Brehmer, and H.-J. Mikeska, ibid. 62, 8903 (2000).
${ }^{4}$ S. Notbohm, P. Ribeiro, B. Lake, D. A. Tennant, K. P. Schmidt, G. S. Uhrig, C. Hess, R. Klingeler, G. Behr, B. Büchner, M. Reehuis, R. I. Bewley, C. D. Frost, P. Manuel, and R. S. Eccleston, Phys. Rev. Lett. 98, 027403 (2007).

5 A. Läuchli, G. Schmid, and M. Troyer, Phys. Rev. B 67, 100409(R) (2003); P. Lecheminant and K. Totsuka, Phys. Rev. B 74, 224426 (2006).

6 T. Hikihara, T. Momoi, and X. Hu, Phys. Rev. Lett. 90, 087204 (2003); V. Gritsev, B. Normand, and D. 
Baeriswyl, Phys. Rev. B 69, 094431 (2004); V. N. Kotov, D. X. Yao, A. H. Castro Neto, and D. K. Campbell, arXiv:0704.0114 1 (unpublished); A. W. Sandvik, Phys. Rev. Lett. 98, 227202 (2007).

7 M. Müller, T. Vekua, and H.-J. Mikeska, Phys. Rev. B 66, 134423 (2002).

${ }^{8}$ K. Takano, K. Kubo, and H. Sakamoto, J. Phys.: Condens. Matter, 8, 6405 (1996); H. Niggemann, G. Uimin, and J. Zittartz, ibid., 9, 9031 (1997); A. Honecker and A. Läuchli, Phys. Rev. B 63, 174407 (2001).

9 K. Okamoto, T. Tonegawa, Y. Takahashi, and M. Kaburagi, J. Phys.: Condens. Matter, 11, 10485 (1999); K. Okamoto, T. Tonegawa, and M. Kaburagi, ibid., 15, 5979 (2003).

${ }^{10}$ H.-J. Mikeska and C. Luckmann, Phys. Rev. B 77, 054405 (2008).

11 C. Vitoriano, M. D. Coutinho-Filho, and E. P. Raposo, J. Phys. A: Math. Gen. 35, 9049 (2002).

12 K. C. Rule, A. U. B. Wolter, S. Süllow, D. A. Tennant, A. Brühl, S. Köhler, B. Wolf, M. Lang, and J. Schreuer, Phys. Rev. Lett. 100, 117202 (2008).

13 N. B. Ivanov and J. Richter, Phys. Lett. A 232, 308 (1997); J. Richter, N. B. Ivanov and J. Schulenburg, J. Phys.: Condens. Matter 10, 3635 (1998); A. Koga, K. Okunishi, and N. Kawakami, Phys. Rev. B 62, 5558 (2000).
14 E. K. Sorensen, S. Eggert, and I. Affleck, J. Phys. A: Math. Gen. 26, 6757 (1993); S. Eggert and I. Affleck, Phys. Rev. B 46, 10866 (1992).

15 S. Sachdev, Quantum phase transitions (University press, Cambridge, 1999).

${ }^{16}$ V. V. Kostyuchenko, I. M. Markevtsev, A. V. Philippov, V. V. Platonov, V. D. Selemir, O. M. Tatsenko, A. K. Zvezdin, and A. Caneschi, Phys. Rev. B 67, 184412 (2003).

17 Interestingly, the multiple-spin exchange term in Eq. (6) also appears in some integrable alternating quantum spin $\left(s_{1}, s_{2}\right)$ chains: see, e.g., G. A. P. Ribeiro and A. Klümper, Nuclear Physics B 801 [FS], 247 (2008), and references therein.

18 E. H. Lieb and D. C. Mattis, J. Math.Phys. 3, 749 (1962).

19 E. Lieb, T. Schultz, and D. Mattis, Ann. Phys. 16, 407 (1961).

20 V. R. Chandra, D. Sen, N. B. Ivanov, and J. Richter, Phys. Rev. B 69, 214406 (2004).

21 N. B. Ivanov and J. Richter, Phys. Rev. B 69, 214420 (2004); S. Yoshikawa and S. Miyashita, J. Phys. Sos. Jpn. 74 Suppl., 71 (2005); K. Hida, J. Phys. Sos. Jpn. 76, 024714 (2007); K. Hida and K. Takano, Phys. Rev. B 78, 064407 (2008). 